\title{
Evidence for the Rare Decay $\Sigma^{+} \rightarrow p \mu^{+} \mu^{-}$
}

\author{
R. Aaij et al. \\ (LHCb Collaboration)
}

(Received 22 December 2017; published 31 May 2018)

\begin{abstract}
A search for the rare decay $\Sigma^{+} \rightarrow p \mu^{+} \mu^{-}$is performed using $p p$ collision data recorded by the LHCb experiment at center-of-mass energies $\sqrt{s}=7$ and $8 \mathrm{TeV}$, corresponding to an integrated luminosity of $3 \mathrm{fb}^{-1}$. An excess of events is observed with respect to the background expectation, with a signal significance of 4.1 standard deviations. No significant structure is observed in the dimuon invariant mass distribution, in contrast with a previous result from the HyperCP experiment. The measured $\Sigma^{+} \rightarrow p \mu^{+} \mu^{-}$ branching fraction is $\left(2.2_{-1.3}^{+1.8}\right) \times 10^{-8}$, where statistical and systematic uncertainties are included, which is consistent with the standard model prediction.
\end{abstract}

DOI: 10.1103/PhysRevLett.120.221803

The $\Sigma^{+} \rightarrow p \mu^{+} \mu^{-}$decay is an $s \rightarrow d$ quark-flavorchanging neutral-current process, allowed only at loop level in the standard model (SM). The process is dominated by long-distance contributions for a predicted branching fraction of $1.6 \times 10^{-8}<\mathcal{B}\left(\Sigma^{+} \rightarrow p \mu^{+} \mu^{-}\right)<9.0 \times 10^{-8}$ [1], while the short-distance SM contributions are suppressed and contribute to the branching fraction at the level of about $10^{-12}$. Evidence for this decay was reported by the HyperCP Collaboration [2] with a measured branching fraction $\mathcal{B}\left(\Sigma^{+} \rightarrow p \mu^{+} \mu^{-}\right)=\left(8.6_{-5.4}^{+6.6} \pm 5.5\right) \times 10^{-8}$, which is compatible with the SM prediction. HyperCP observed three candidates; remarkably, all of them have almost the same dimuon invariant mass of $m_{X^{0}}=214.3 \pm 0.5 \mathrm{MeV} / c^{2}$, close to the lower kinematic limit. Such a distribution, if confirmed, would point towards a process with an intermediate particle $X^{0}$ coming from the $\Sigma^{+}$baryon and decaying into two muons, i.e., a $\Sigma^{+} \rightarrow p X^{0}\left(\rightarrow \mu^{+} \mu^{-}\right)$ decay, which would constitute evidence for physics beyond the SM (BSM). Various BSM theories have been proposed to explain the HyperCP result. The intermediate $X^{0}$ particle could be, for example, a light pseudoscalar Higgs boson $[3,4]$ or a sgoldstino $[5,6]$ in various supersymmetric models. Other interpretations and implications can be found in Refs. [7-13]; in general a pseudoscalar particle is favored over a scalar particle and a lifetime of the order of $10^{-14} \mathrm{~s}$ is estimated for the former case. Attempts to confirm the existence of this $X^{0}$ particle have been made at several experiments in various initial and final states without finding

\footnotetext{
*Full author list given at the end of the article.

Published by the American Physical Society under the terms of the Creative Commons Attribution 4.0 International license. Further distribution of this work must maintain attribution to the author(s) and the published article's title, journal citation, and DOI. Funded by SCOAP.
}

any signal [14-21]; these null results include studies of the decays $B_{(s)}^{0} \rightarrow \mu^{+} \mu^{-} \mu^{+} \mu^{-}$[22], $B^{0} \rightarrow K^{* 0} \mu^{+} \mu^{-}$[23], $B^{+} \rightarrow$ $K^{+} \mu^{+} \mu^{-}$[24], and a search for photonlike particles [25] by the $\mathrm{LHCb}$ experiment. However, the search for the $\Sigma^{+} \rightarrow$ $p \mu^{+} \mu^{-}$decay has not been repeated due to the lack of experiments with large hyperon production rates and to the experimental difficulty of reconstructing soft and long-lived hadrons.

Hyperons are produced copiously in high-energy protonproton collisions at the Large Hadron Collider. A search for $\Sigma^{+} \rightarrow p \mu^{+} \mu^{-}$decays at the LHCb experiment, as also suggested in Ref. [26], could therefore confirm or disprove the HyperCP evidence, and the branching fraction can be measured. This Letter presents a search for the $\Sigma^{+} \rightarrow$ $p \mu^{+} \mu^{-}$decay performed using $p p$ collision data recorded by the LHCb experiment at center-of-mass energies $\sqrt{s}=$ 7 and $8 \mathrm{TeV}$, corresponding to an integrated luminosity of $3 \mathrm{fb}^{-1}$. The inclusion of charge-conjugated processes is implied throughout this Letter.

This search follows a strategy similar to that of other studies of rare decays in $\mathrm{LHCb}$, although with differences due to the relatively low transverse momenta of the finalstate particles. First, a loose selection is applied based on geometric and kinematic variables. The final sample is obtained rejecting the background with requirements on the output of a multivariate selection, based on a boosted decision tree algorithm (BDT) [27,28], and on particle identification variables. The signal yield is obtained from a fit to the $p \mu^{+} \mu^{-}$invariant-mass spectrum and is converted into a branching fraction by normalizing to the $\Sigma^{+} \rightarrow p \pi^{0}$ control channel. The analysis is designed in order to search for possible peaks in the dimuon invariant-mass distribution, in view of the possible existence of unknown intermediate particles.

The $\mathrm{LHCb}$ detector is a single-arm forward spectrometer covering the pseudorapidity range $2<\eta<5$, described in 
detail in Refs. [29,30]. It includes a high-precision tracking system consisting of a silicon-strip vertex detector surrounding the $p p$ interaction region, a large-area siliconstrip detector located upstream of a dipole magnet with a bending power of about $4 \mathrm{Tm}$, and three stations of siliconstrip detectors and straw drift tubes placed downstream of the magnet. Particle identification is provided by two ringimaging Cherenkov detectors, an electromagnetic and a hadronic calorimeter, and a muon system composed of alternating layers of iron and multiwire proportional chambers.

The online event selection is performed by a trigger system, which consists of a hardware stage, based on information from the calorimeter and muon systems, followed by two software stages. The first software stage performs a preliminary event reconstruction based on partial information while the second applies a full event reconstruction. Each of the three trigger stages is divided into many trigger selections dedicated to various types of signals. The final-state particles from the signal decay involved in this analysis typically have insufficient transverse momenta to satisfy the requirements of one or more trigger stages. Nevertheless, given the large production rate of $\Sigma^{+}$baryons in $p p$ collisions, the present search can be performed with data selected at one or more trigger stages by other particles in the event. In the offline processing, trigger decisions are associated with reconstructed candidates. A trigger decision can thus be ascribed to the reconstructed candidate, the rest of the event or a combination of both; events triggered as such are defined respectively as triggered on signal (TOS), triggered independently of signal (TIS), and triggered on both. While all the candidates passing the trigger selection are used in the search for $\Sigma^{+} \rightarrow p \mu^{+} \mu^{-}$decays, only the TIS candidates are used in the normalization channel $\Sigma^{+} \rightarrow p \pi^{0}$. Furthermore, control channels with large yields are exploited to estimate the trigger efficiency by measuring the overlap of candidates which are TIS and TOS simultaneously [31].

Simulation is used to devise and optimize the analysis strategy, as well as to estimate reconstruction and selection efficiencies. In the simulation, $p p$ collisions are generated using PythIA [32] with a specific LHCb configuration [33]. Decays of hadronic particles are described by EvTGEN [34], in which final-state radiation is generated using Рнотоs [35]. The interaction of the generated particles with the detector, and its response, are implemented using the GEANT4 toolkit [36], as described in Ref. [37]. The signal $\Sigma^{+} \rightarrow p \mu^{+} \mu^{-}$decay is generated according to a phase-space model.

Candidate $\Sigma^{+} \rightarrow p \mu^{+} \mu^{-}$decays are selected by combining two good-quality oppositely charged tracks identified as muons with a third track identified as a proton. The three tracks are required to form a secondary vertex (SV) with a good vertex-fit quality. The short lifetime estimated for the
$X^{0}$ particle would result in a prompt signal in this search; hence, no attempt is made to distinguish the dimuon origin vertex from the SV of the $\Sigma^{+}$baryon. The measured $\Sigma^{+}$ candidate proper decay time is required to be greater than $6 \mathrm{ps}$, ensuring that the $\mathrm{SV}$ is displaced from any $p p$ interaction vertex (primary vertex, PV). The final-state particles are required to be inconsistent with originating from any PV in the event. Only $\Sigma^{+}$candidates with transverse momentum $p_{T}>0.5 \mathrm{GeV} / c$ and a decay topology consistent with a particle originating from the PV are retained. A candidate $\Sigma^{+} \rightarrow p \mu^{+} \mu^{-}$decay is considered only if its invariant mass, $m_{p \mu^{+} \mu^{-}}$, satisfies $\left|m_{p \mu^{+} \mu^{-}}-m_{\Sigma^{+}}\right|<500 \mathrm{MeV} / c^{2}$, where $m_{\Sigma^{+}}$is the known mass of the $\Sigma^{+}$particle [38]. The background component due to $\Lambda \rightarrow p \pi^{-}$decays is vetoed by discarding candidates having a $p \mu^{-}$pair invariant mass, calculated with the $p \pi^{-}$ mass hypothesis, within $10 \mathrm{MeV} / c^{2}$ from the known $\Lambda$ mass [38]. Possible backgrounds from decays peaking in the $p \mu^{+} \mu^{-}$invariant mass have been examined, including $K^{+} \rightarrow \pi^{+} \pi^{-} \pi^{+}, K^{+} \rightarrow \pi^{+} \mu^{-} \mu^{+}$, and various hyperon decays, and none has been found to contribute significantly. After all selection requirements, no retained event contains more than one candidate.

Candidate $\Sigma^{+} \rightarrow p \pi^{0}$ decays are selected by combining one good-quality track identified as a proton with a $\pi^{0}$ reconstructed in the $\pi^{0} \rightarrow \gamma \gamma$ mode from two clusters in the electromagnetic calorimeter. Given the impossibility to reconstruct the $\Sigma^{+}$decay SV with the proton track only, the momentum direction of the $\pi^{0}$ is calculated assuming the $\pi^{0}$ is produced at the PV. The selection of $\Sigma^{+} \rightarrow p \pi^{0}$ decays is similar to that of the signal, with tighter requirements applied, in order to reduce the large combinatorial background, on the proton identification and on the transverse momenta of the final-state particles $\left(p_{T}>0.5 \mathrm{GeV} / c\right.$ for the proton and $p_{T}>0.7 \mathrm{GeV} / c$ for the $\pi^{0}$ ). Finally, candidate $K^{+} \rightarrow \pi^{+} \pi^{-} \pi^{+}$decays, selected as control channel for various parts of the analysis, are required to pass a selection similar to that of the signal, starting from three good-quality tracks, with total charge equal to \pm 1 , and which are assigned the pion mass hypothesis without requirements on the identification of the particle.

The sample of $\Sigma^{+} \rightarrow p \mu^{+} \mu^{-}$candidates in data after the initial selection is dominated by combinatorial background, part of which is due to misidentified particles. This background is rejected by placing requirements on the BDT output variable and on multivariate particle identification variables [30] on the muons and on the proton. The BDT combines information from the following input variables: the angle between the $\Sigma^{+}$reconstructed momentum and the vector joining the $\mathrm{PV}$ to the $\mathrm{SV}$, the flight distance significance of the $\Sigma^{+}$candidate, the distance of closest approach among the final-state particles, the transverse momenta of the final-state particles, the impact parameter $\chi^{2}\left(\chi_{\mathrm{IP}}^{2}\right)$ of the final-state particles, defined as the difference 
between the vertex-fit $\chi^{2}$ of a PV formed with and without the particle in question, the $\chi_{\mathrm{IP}}^{2}$ of the $\Sigma^{+}$candidate, the $\chi^{2}$ of the SV, and an isolation variable constructed from the number of tracks within an angular cone around each of the final-state particles. These variables are chosen so that the dependence on the $p \mu^{+} \mu^{-}$invariant mass and on the dimuon invariant mass is small and linear to minimize potential biases. The BDT is optimized using simulated samples of $\Sigma^{+} \rightarrow p \mu^{+} \mu^{-}$events for the signal and $\bar{p} \mu^{+} \mu^{+}$ candidates in data for the background. The selection for the control $\bar{p} \mu^{+} \mu^{+}$sample is identical to that of the signal but considering muons of identical charge. The final selection criteria are chosen in order to optimize the potential to obtain evidence for a signal with a branching fraction as small as possible [39]. No BDT selection is applied to the normalization and control channels.

The number of signal candidates is converted into a branching fraction with the formula

$$
\begin{aligned}
\mathcal{B}\left(\Sigma^{+} \rightarrow p \mu^{+} \mu^{-}\right) & =\frac{\varepsilon_{\Sigma^{+} \rightarrow p \pi^{0}}}{\varepsilon_{\Sigma^{+} \rightarrow p \mu^{+} \mu^{-}}} \frac{N_{\Sigma^{+} \rightarrow p \mu^{+} \mu^{-}}}{N_{\Sigma^{+} \rightarrow p \pi^{0}}} \mathcal{B}\left(\Sigma^{+} \rightarrow p \pi^{0}\right) \\
& =\alpha N_{\Sigma^{+} \rightarrow p \mu^{+} \mu^{-}},
\end{aligned}
$$

where $\varepsilon, N$, and $\mathcal{B}$ are the efficiency, candidate yield, and branching fraction of the corresponding channel, respectively, and $\alpha$ is the single-event sensitivity. The ratio of signal and normalization channel efficiencies, which includes the acceptance, the trigger efficiency, the reconstruction efficiency of the final-state particles, and the selection efficiency, is computed with samples of simulated events corrected to take into account known differences between data and simulation. The reconstruction efficiency for the $\pi^{0}$ is calibrated using the ratio of $B^{+} \rightarrow J / \psi K^{*+}\left(\rightarrow K^{+} \pi^{0}\right)$ and $B^{+} \rightarrow J / \psi K^{+}$ decays reconstructed in data [40]. The particle-identification efficiencies of protons and muons are calibrated with control channels in data. Residual differences between data and simulation are treated as sources of systematic uncertainty. The ratio of the trigger efficiencies for the signal and normalization channels is estimated with simulated samples and cross-checked in data: the trigger efficiency is obtained for selected trigger lines from the overlap of TIS and TOS events in the normalization channel and is compared between data and simulation [31]. The small size of this overlap induces a $40 \%$ relative systematic uncertainty associated with the trigger efficiency ratio. The ratio of the trigger efficiencies is of the order of 0.09 , owing to the use of all events for the signal, while TIS-only events are used for the normalization channel. Possible differences in the BDT selection efficiency for the $\Sigma^{+} \rightarrow p \mu^{+} \mu^{-}$signal in data and in simulation are calibrated using the $K^{+} \rightarrow$ $\pi^{+} \pi^{-} \pi^{+}$control channel. The sources of systematic uncertainties associated with the normalization are reported in Table I.
TABLE I. Relative systematic uncertainties associated with the normalization.

\begin{tabular}{lr}
\hline \hline Source & Uncertainty \\
\hline Selection efficiency & $1 \%$ \\
BDT efficiency & $6 \%$ \\
PID efficiency ratio & $28 \%$ \\
$\pi^{0}$ efficiency & $10 \%$ \\
Trigger efficiency ratio & $40 \%$ \\
Total & $50 \%$ \\
\hline \hline
\end{tabular}

The observed number of $\Sigma^{+} \rightarrow p \pi^{0}$ candidates is $(1171 \pm 9) \times 10^{3}$, as obtained from a binned extended maximum likelihood fit to the corrected invariant mass distribution $m_{\Sigma}^{\text {corr }}$. The corrected invariant mass is defined as $m_{\Sigma}^{\text {corr }}=m_{p \gamma \gamma}-m_{\gamma \gamma}+m_{\pi^{0}}$, where $m_{\pi^{0}}$ is the known mass of the $\pi^{0}$ meson [38], to account for the limited precision in the reconstructed invariant mass of the two photons $\left(m_{\gamma \gamma}\right)$. The $\Sigma^{+} \rightarrow p \pi^{0}$ distribution is described as a Gaussian function with a power-law tail on the higher-mass side, while the background is described by a modified ARGUS function [41], where the power parameter is allowed to vary as in Ref. [42]. The distribution is shown in Fig. 1, superimposed with the fit.

The single-event sensitivity is $\alpha=(2.2 \pm 1.2) \times 10^{-9}$, where the uncertainty is dominated by the systematic contribution. This sensitivity corresponds to about $10^{14}$ $\Sigma^{+}$baryons produced in the $\mathrm{LHCb}$ acceptance in the considered data set. The number of expected signal $\Sigma^{+} \rightarrow$ $p \mu^{+} \mu^{-}$candidates is $23 \pm 20$ assuming a branching fraction of $(5 \pm 4) \times 10^{-8}$, to cover the range predicted by the SM.

The observed number of signal $\Sigma^{+} \rightarrow p \mu^{+} \mu^{-}$decays is obtained with a fit to the $p \mu^{+} \mu^{-}$invariant-mass distribution in the range $1149.6<m_{p \mu^{+} \mu^{-}}<1409.6 \mathrm{MeV} / c^{2}$. The signal distribution is described by an Hypatia function

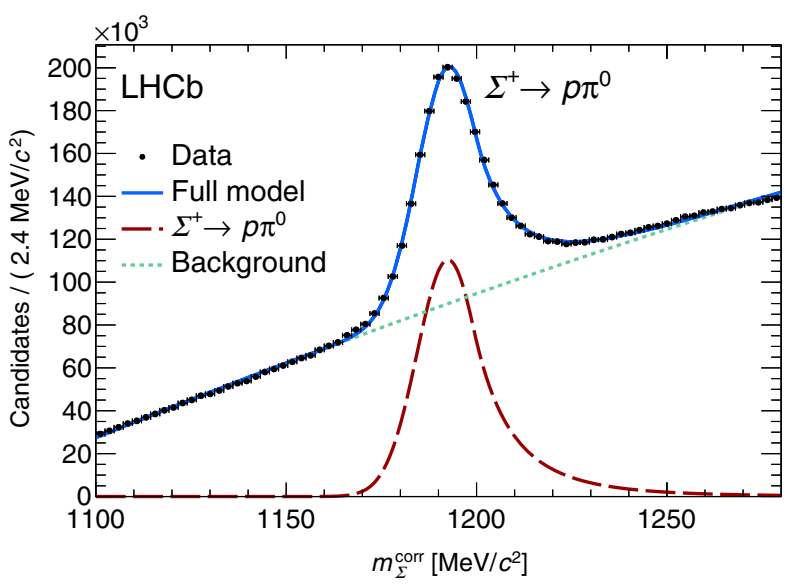

FIG. 1. Distribution of the corrected mass $m_{\Sigma}^{\text {corr }}$, defined as in the text, for $\Sigma^{+} \rightarrow p \pi^{0}$ candidates superimposed with the fit to data. 


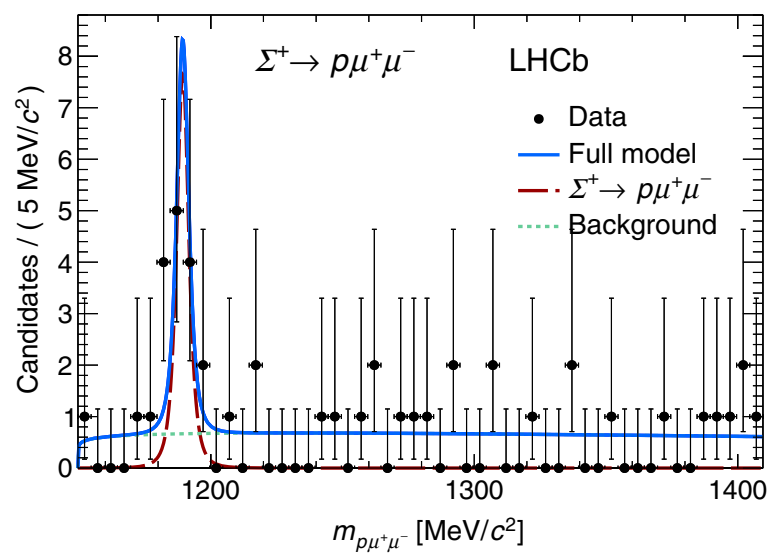

FIG. 2. Invariant mass distribution of $\Sigma^{+} \rightarrow p \mu^{+} \mu^{-}$candidates in data.

[43]. The peak position and resolution are calibrated using the control channel $K^{+} \rightarrow \pi^{+} \pi^{-} \pi^{+}$and by comparing distributions in data and simulation. No bias is seen in the peak position, while a relative positive correction of $25 \%$ with respect to the simulation is applied to the resolution. A resolution of $4.28 \pm 0.19 \mathrm{MeV} / c^{2}$ is obtained for the signal $\Sigma^{+} \rightarrow p \mu^{+} \mu^{-}$distribution and is used in the fit to define a Gaussian constraint to the width of the signal distribution. The combinatorial background is described as a modified ARGUS function with all parameters left free with the exception of the threshold, which is fixed to the kinematic limit. The shape of this background is also cross-checked with that of $\bar{p} \mu^{+} \mu^{+}$candidates in data.

The invariant mass distribution of the $\Sigma^{+} \rightarrow p \mu^{+} \mu^{-}$ candidates in data is shown in Fig. 2. The significance of the signal is $4.1 \sigma$, obtained from a comparison of the likelihood value of the nominal fit with that of a background-only fit [44], and with the relevant systematic uncertainties included as Gaussian constraints to the likelihood. A signal yield of $10.2_{-3.5}^{+3.9}$ is observed. The corresponding branching fraction is $\mathcal{B}\left(\Sigma^{+} \rightarrow p \mu^{+} \mu^{-}\right)=$ $\left(2.2_{-0.8}^{+0.9}{ }_{-1.1}^{+1.5}\right) \times 10^{-8}$, where the first uncertainty is statistical and the second is systematic, consistent with the SM prediction. As a cross-check, the fit is repeated with tighter or looser requirements on the BDT or on the particle identification variables, and the signal yield is found to vary consistently with the signal efficiency. The fit is also repeated assuming a linear function for the background, in place of an ARGUS function, and the signal yield and significance are found to be stable. Candidates in data are composed of about $48 \% \Sigma^{+}$antibaryons in the final sample.

The distribution of the dimuon invariant mass after background subtraction, performed with the sPlot method [45], is shown in Fig. 3. A scan for a possible resonant structure in the dimuon invariant mass is performed, considering a region within two times the resolution in the $p \mu^{+} \mu^{-}$invariant mass around the known $\Sigma^{+}$mass. The

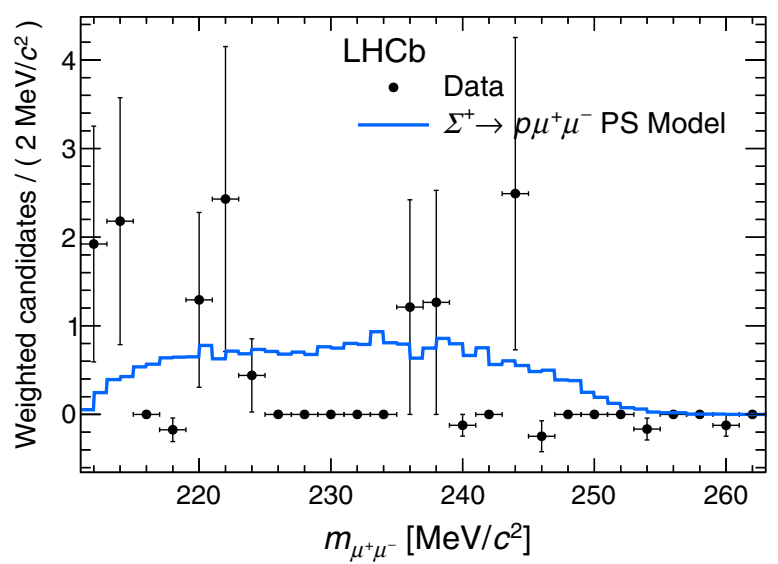

FIG. 3. Background-subtracted distribution of the dimuon invariant mass for $\Sigma^{+} \rightarrow p \mu^{+} \mu^{-}$candidates, superimposed with the distribution from the simulated phase-space (PS) model. Uncertainties on data points are calculated as the square root of the sum of squared weights.

distribution of these candidates as a function of the dimuon invariant mass is shown in the Supplemental Material [46]. Steps of half the resolution on the dimuon invariant mass, $\sigma\left(m_{\mu^{+} \mu^{-}}\right)$, are considered in this scan, following the method outlined in Ref. [47]. The value of $\sigma\left(m_{\mu^{+} \mu^{-}}\right)$varies in the range $[0.3,2.3] \mathrm{MeV} / c^{2}$ depending on the dimuon invariant mass as shown in Ref. [46]. For each step the putative signal is estimated in a window of $\pm 1.5 \times \sigma\left(m_{\mu^{+} \mu^{-}}\right)$around the considered particle mass, while the background is estimated from the lower and upper sidebands contained in the range $[1.5-4.0] \times \sigma\left(m_{\mu^{+} \mu^{-}}\right)$from the same mass. Only one of the two sidebands is considered when the second is outside the allowed kinematic range. The local $p$-value of the background-only hypothesis as a function of the dimuon mass is shown in Ref. [46], and no significant signal is found. The fit to the $p \mu^{+} \mu^{-}$invariant mass is then repeated restricting the sample to events within 1.5 times the resolution from the putative particle $\left(m_{\mu^{+} \mu^{-}} \in[214.3 \pm 0.75] \mathrm{MeV} / c^{2}\right)$. No significant signal is found and a yield of $3.0_{-1.4}^{+1.7}$ is measured corresponding to $30 \%$ of the $\Sigma^{+} \rightarrow p \mu^{+} \mu^{-}$yield. An upper limit on the branching fraction of the resonant channel is thus set with the $\mathrm{CL}_{S}$ method [48] at $\mathcal{B}\left(\Sigma^{+} \rightarrow p X^{0}\left(\rightarrow \mu^{+} \mu^{-}\right)\right)<1.4 \times$ $10^{-8}\left(1.7 \times 10^{-8}\right)$ at $90 \%(95 \%)$ confidence level.

In summary, a search for the $\Sigma^{+} \rightarrow p \mu^{+} \mu^{-}$rare decay is performed by the $\mathrm{LHCb}$ experiment using $p p$ collisions at center-of-mass energies $\sqrt{s}=7$ and $8 \mathrm{TeV}$, corresponding to an integrated luminosity of $3 \mathrm{fb}^{-1}$. Evidence for the $\Sigma^{+} \rightarrow p \mu^{+} \mu^{-}$decay is found with a significance of 4.1 standard deviations, including systematic uncertainties. A branching fraction $\mathcal{B}\left(\Sigma^{+} \rightarrow p \mu^{+} \mu^{-}\right)=\left(2.2_{-1.3}^{+1.8}\right) \times 10^{-8}$ is measured, consistent with the SM prediction. No significant peak consistent with an intermediate particle is found in the dimuon invariant-mass distribution of the signal candidates. 
We express our gratitude to our colleagues in the CERN accelerator departments for the excellent performance of the LHC. We thank the technical and administrative staff at the LHCb institutes. We acknowledge support from CERN and from the national agencies: CAPES, CNPq, FAPERJ and FINEP (Brazil); MOST and NSFC (China); CNRS/IN2P3 (France); BMBF, DFG and MPG (Germany); INFN (Italy); NWO (Netherlands); MNiSW and NCN (Poland); MEN/ IFA (Romania); MinES and FASO (Russia); MinECo (Spain); SNSF and SER (Switzerland); NASU (Ukraine); STFC (United Kingdom); NSF (USA). We acknowledge the computing resources that are provided by CERN, IN2P3 (France), KIT and DESY (Germany), INFN (Italy), SURF (Netherlands), PIC (Spain), GridPP (United Kingdom), RRCKI and Yandex LLC (Russia), CSCS (Switzerland), IFIN-HH (Romania), CBPF (Brazil), PL-GRID (Poland) and OSC (USA). We are indebted to the communities behind the multiple open-source software packages on which we depend. Individual groups or members have received support from AvH Foundation (Germany), EPLANET, Marie Skłodowska-Curie Actions and ERC (European Union), ANR, Labex P2IO and OCEVU, and Région AuvergneRhône-Alpes (France), RFBR, RSF and Yandex LLC (Russia), GVA, XuntaGal and GENCAT (Spain), Herchel Smith Fund, the Royal Society, the English-Speaking Union and the Leverhulme Trust (United Kingdom).

[1] X.-G. He, J. Tandean, and G. Valencia, Decay $\Sigma^{+} \rightarrow p \ell^{+} \ell^{-}$ within the standard model, Phys. Rev. D 72, 074003 (2005).

[2] H. Park et al. (HyperCP Collaboration), Evidence for the Decay $\Sigma^{+} \rightarrow p \mu^{+} \mu^{-}$, Phys. Rev. Lett. 94, 021801 (2005).

[3] X.-G. He, J. Tandean, and G. Valencia, Does the HyperCP Evidence for the Decay $\Sigma^{+} \rightarrow p \mu^{+} \mu^{-}$Indicate a Light Pseudoscalar Higgs Boson?, Phys. Rev. Lett. 98, 081802 (2007).

[4] X.-G. He, J. Tandean, and G. Valencia, Light Higgs production in hyperon decay, Phys. Rev. D 74, 115015 (2006).

[5] D. S. Gorbunov and V. A. Rubakov, Sgoldstino interpretation of HyperCP events, Phys. Rev. D 73, 035002 (2006).

[6] S. V. Demidov and D. S. Gorbunov, More about the sgoldstino interpretation of HyperCP events, JETP Lett. 84, 479 (2007).

[7] X.-G. He, J. Tandean, and G. Valencia, Implications of a new particle from the HyperCP data on $\Sigma^{+} \rightarrow p \mu^{+} \mu^{-}$, Phys. Lett. B 631, 100 (2005).

[8] C. Q. Geng and Y. K. Hsiao, Constraints on the new particle in $\Sigma^{+} \rightarrow p \mu^{+} \mu^{-}$, Phys. Lett. B 632, 215 (2006).

[9] N. G. Deshpande, G. Eilam, and J. Jiang, On the possibility of a new boson $X^{0}(214 \mathrm{MeV})$ in $\Sigma^{+} \rightarrow p \mu^{+} \mu^{-}$, Phys. Lett. B 632, 212 (2006).

[10] C.-H. Chen, C.-Q. Geng, and C.-W. Kao, U-boson and the HyperCP exotic events, Phys. Lett. B 663, 400 (2008).

[11] G. Xiangdong, C. S. Li, Z. Li, and H. Zhang, Contributions from SUSY-FCNC couplings to the interpretation of the HyperCP events for the decay $\Sigma^{+} \rightarrow p \mu^{+} \mu^{-}$, Eur. Phys. J. C 55, 317 (2008).
[12] M. L. Mangano and P. Nason, Radiative quarkonium decays and the NMSSM Higgs interpretation of the HyperCP $\Sigma^{+} \rightarrow$ $p \mu^{+} \mu^{-}$events, Mod. Phys. Lett. A 22, 1373 (2007).

[13] M. Pospelov, Secluded U(1) below the weak scale, Phys. Rev. D 80, 095002 (2009).

[14] W. Love et al. (CLEO Collaboration), Search for Very Light CP-Odd Higgs Boson in Radiative Decays of $\Upsilon(1 S)$, Phys. Rev. Lett. 101, 151802 (2008).

[15] Y. C. Tung et al. (E391a Collaboration), Search for a Light Pseudoscalar Particle in the Decay $K_{L}^{0} \rightarrow \pi^{0} \pi^{0} X$, Phys. Rev. Lett. 102, 051802 (2009).

[16] V. M. Abazov et al. (D0 Collaboration), Search for Next-toMinimal Supersymmetric Higgs Bosons in the $h \rightarrow a a \rightarrow$ $\mu \mu \mu \mu, \mu \mu \tau \tau$ Channels Using $p \bar{p}$ Collisions at $\sqrt{s}=$ 1.96 TeV, Phys. Rev. Lett. 103, 061801 (2009).

[17] B. Aubert et al. (BABAR Collaboration), Search for Dimuon Decays of a Light Scalar Boson in Radiative Transitions $\Upsilon \rightarrow \gamma A^{0}$, Phys. Rev. Lett. 103, 081803 (2009).

[18] H. J. Hyun et al. (Belle Collaboration), Search for a Low Mass Particle Decaying into $\mu^{+} \mu^{-}$in $B^{0} \rightarrow K^{* 0} X$ and $B^{0} \rightarrow$ $\rho^{0} X$ at Belle, Phys. Rev. Lett. 105, 091801 (2010).

[19] E. Abouzaid et al. (KTeV Collaboration), Search for the Rare Decays $K_{L} \rightarrow \pi^{0} \pi^{0} \mu^{+} \mu^{-}$and $K_{L} \rightarrow \pi^{0} \pi^{0} X^{0} \rightarrow$ $\pi^{0} \pi^{0} \mu^{+} \mu^{-}$, Phys. Rev. Lett. 107, 201803 (2011).

[20] J. P. Lees et al. (BABAR Collaboration), Search for a Dark Photon in $e^{+} e^{-}$Collisions at BABAR, Phys. Rev. Lett. 113, 201801 (2014).

[21] M. Ablikim (BESIII Collaboration), Search for a light $C P$-odd Higgs boson in radiative decays of $J / \psi$, Phys. Rev. D 93, 052005 (2016).

[22] R. Aaij et al. (LHCb Collaboration), Search for Rare $B_{(s)}^{0} \rightarrow \mu^{+} \mu^{-} \mu^{+} \mu^{-}$Decays, Phys. Rev. Lett. 110, 211801 (2013).

[23] R. Aaij et al. (LHCb Collaboration), Search for HiddenSector Bosons in $B^{0} \rightarrow K^{* 0} \mu^{+} \mu^{-}$Decays, Phys. Rev. Lett. 115, 161802 (2015).

[24] R. Aaij et al. (LHCb Collaboration), Search for long-lived scalar particles in $\mathcal{B}^{+} \rightarrow K^{+} \chi\left(\mu^{+} \mu^{-}\right)$decays, Phys. Rev. D 95, 071101 (2017).

[25] R. Aaij et al. (LHCb Collaboration), Search for Dark Photons Produced in $13 \mathrm{TeV} p p$ Collisions, Phys. Rev. Lett. 120, 061801 (2018).

[26] H.-K. Park, Search for the HyperCP event at the LHCb experiment, J. High Energy Phys. 10 (2010) 052.

[27] L. Breiman, J. H. Friedman, R. A. Olshen, and C. J. Stone, Classification and Regression Trees (Wadsworth International Group, Belmont, CA, 1984).

[28] Y. Freund and R. E. Schapire, A decision-theoretic generalization of on-line learning and an application to boosting, J. Comput. Syst. Sci. 55, 119 (1997).

[29] A. A. Alves Jr. et al. (LHCb Collaboration), The LHCb detector at the LHC, J. Instrum. 3, S08005 (2008).

[30] R. Aaij et al. (LHCb Collaboration), LHCb detector performance, Int. J. Mod. Phys. A 30, 1530022 (2015).

[31] R. Aaij et al. (LHCb Collaboration), The LHCb trigger and its performance in 2011, J. Instrum. 8, P04022 (2013).

[32] T. Sjöstrand, S. Mrenna, and P. Skands, PYTHIA 6.4 physics and manual, J. High Energy Phys. 05 (2006) 026; A brief introduction to PYTHIA 8.1, Comput. Phys. Commun. 178, 852 (2008). 
[33] I. Belyaev et al., Handling of the generation of primary events in Gauss, the LHCb simulation framework, J. Phys. Conf. Ser. 331, 032047 (2011).

[34] D. J. Lange, The EvtGen particle decay simulation package, Nucl. Instrum. Methods Phys. Res., Sect. A 462, 152 (2001).

[35] P. Golonka and Z. Was, PHOTOS Monte Carlo: A precision tool for QED corrections in $Z$ and $W$ decays, Eur. Phys. J. C 45, 97 (2006).

[36] J. Allison et al. (Geant4 Collaboration), Geant4 developments and applications, IEEE Trans. Nucl. Sci. 53, 270 (2006); S. Agostinelli et al. (Geant4 Collaboration), Geant4: A simulation toolkit, Nucl. Instrum. Methods Phys. Res., Sect. A 506, 250 (2003).

[37] M. Clemencic, G. Corti, S. Easo, C. R. Jones, S. Miglioranzi, M. Pappagallo, and P. Robbe, The LHCb simulation application, Gauss: Design, evolution and experience, J. Phys. Conf. Ser. 331, 032023 (2011).

[38] C. Patrignani et al. (Particle Data Group), Review of particle physics, Chin. Phys. C 40, 100001 (2016).

[39] G. Punzi, Sensitivity of searches for new signals and its optimization, arXiv:physics/0308063.

[40] E. Govorkova, Study of $\pi^{0} / \gamma$ efficiency using $B$ meson decays in the LHCb experiment, Phys. At. Nucl. 79, 1474 (2016).
[41] H. Albrecht et al. (ARGUS Collaboration), Search for hadronic $b \rightarrow u$ decays, Phys. Lett. B 241, 278 (1990).

[42] S. Dobbs et al. (CLEO Collaboration), Measurement of absolute hadronic branching fractions of D mesons and $e^{+} e^{-} \rightarrow D \bar{D}$ cross sections at the $\psi(3770)$, Phys. Rev. D 76, 112001 (2007).

[43] D. M. Santos and F. Dupertuis, Mass distributions marginalized over per-event errors, Nucl. Instrum. Methods Phys. Res., Sect. A 764, 150 (2014).

[44] S. S. Wilks, The large-sample distribution of the likelihood ratio for testing composite hypotheses, Ann. Math. Stat. 9, 60 (1938).

[45] M. Pivk and F. R. Le Diberder, sPlot: A statistical tool to unfold data distributions, Nucl. Instrum. Methods Phys. Res., Sect. A 555, 356 (2005).

[46] See Supplemental Material at http://link.aps.org/ supplemental/10.1103/PhysRevLett.120.221803 for additional plots and tables.

[47] M. Williams, Searching for a particle of unknown mass and lifetime in the presence of an unknown non-monotonic background, J. Instrum. 10, P06002 (2015).

[48] A. L. Read, Presentation of search results: The $\mathrm{CL}_{S}$ technique, J. Phys. G 28, 2693 (2002).

R. Aaij, ${ }^{40}$ B. Adeva,${ }^{39}$ M. Adinolfi, ${ }^{48}$ Z. Ajaltouni, ${ }^{5}$ S. Akar, ${ }^{59}$ J. Albrecht,${ }^{10}$ F. Alessio,${ }^{40}$ M. Alexander, ${ }^{53}$ A. Alfonso Albero, ${ }^{38}$ S. Ali, ${ }^{43}$ G. Alkhazov, ${ }^{31}$ P. Alvarez Cartelle, ${ }^{55}$ A. A. Alves Jr., ${ }^{59}$ S. Amato, ${ }^{2}$ S. Amerio, ${ }^{23}$ Y. Amhis, ${ }^{7}$ L. An, ${ }^{3}$ L. Anderlini, ${ }^{18}$ G. Andreassi, ${ }^{41}$ M. Andreotti, ${ }^{17, a}$ J. E. Andrews,${ }^{60}$ R. B. Appleby, ${ }^{56}$ F. Archilli, ${ }^{43}$ P. d'Argent, ${ }^{12}$ J. Arnau Romeu, ${ }^{6}$ A. Artamonov, ${ }^{37}$ M. Artuso, ${ }^{61}$ E. Aslanides, ${ }^{6}$ M. Atzeni, ${ }^{42}$ G. Auriemma, ${ }^{26}$ M. Baalouch, ${ }^{5}$ I. Babuschkin, ${ }^{56}$ S. Bachmann, ${ }^{12}$ J. J. Back, ${ }^{50}$ A. Badalov, ${ }^{38, b}$ C. Baesso, ${ }^{62}$ S. Baker,${ }^{55}$ V. Balagura, ${ }^{7, c}$ W. Baldini, ${ }^{17}$ A. Baranov, ${ }^{35}$ R. J. Barlow,${ }^{56}$ C. Barschel, ${ }^{40}$ S. Barsuk, ${ }^{7}$ W. Barter, ${ }^{56}$ F. Baryshnikov, ${ }^{32}$ V. Batozskaya,${ }^{29}$ V. Battista,${ }^{41}$ A. Bay ${ }^{41}$ L. Beaucourt ${ }^{4}$ J. Beddow ${ }^{53}$ F. Bedeschi, ${ }^{24}$ I. Bediaga, ${ }^{1}$ A. Beiter,${ }^{61}$ L. J. Bel, ${ }^{43}$ N. Beliy,${ }^{63}$ V. Bellee, ${ }^{41}$ N. Belloli, ${ }^{21, d}$ K. Belous, ${ }^{37}$ I. Belyaev, ${ }^{32,40}$ E. Ben-Haim, ${ }^{8}$ G. Bencivenni, ${ }^{19}$ S. Benson, ${ }^{43}$ S. Beranek, ${ }^{9}$ A. Berezhnoy,${ }^{33}$ R. Bernet, ${ }^{42}$ D. Berninghoff, ${ }^{12}$ E. Bertholet, ${ }^{8}$ A. Bertolin, ${ }^{23}$ C. Betancourt, ${ }^{42}$ F. Betti, ${ }^{15}$ M. O. Bettler, ${ }^{40}$ M. van Beuzekom, ${ }^{43}$ Ia. Bezshyiko, ${ }^{42}$ S. Bifani, ${ }^{47}$ P. Billoir, ${ }^{8}$ A. Birnkraut, ${ }^{10}$ A. Bizzeti, ${ }^{18, e}$ M. Bjørn,${ }^{57}$ T. Blake, ${ }^{50}$ F. Blanc, ${ }^{41}$ S. Blusk,${ }^{61}$ V. Bocci, ${ }^{26}$ T. Boettcher, ${ }^{58}$ A. Bondar, ${ }^{36, f}$ N. Bondar, ${ }^{31}$ I. Bordyuzhin, ${ }^{32}$ S. Borghi, ${ }^{56,40}$ M. Borisyak, ${ }^{35}$ M. Borsato ${ }^{39}$ F. Bossu, ${ }^{7}$ M. Boubdir, ${ }^{9}$ T. J. V. Bowcock, ${ }^{54}$ E. Bowen, ${ }^{42}$ C. Bozzi, ${ }^{17,40}$ S. Braun, ${ }^{12}$ J. Brodzicka, ${ }^{27}$ D. Brundu, ${ }^{16}$ E. Buchanan, ${ }^{48}$ C. Burr, ${ }^{56}$ A. Bursche, ${ }^{16,9}$ J. Buytaert, ${ }^{40}$ W. Byczynski, ${ }^{40}$ S. Cadeddu, ${ }^{16}$ H. Cai, ${ }^{64}$ R. Calabrese, ${ }^{17, a}$ R. Calladine, ${ }^{47}$ M. Calvi, ${ }^{21, d}$ M. Calvo Gomez ${ }^{38, b}$ A. Camboni, ${ }^{38, b}$ P. Campana, ${ }^{19}$ D. H. Campora Perez, ${ }^{40}$ L. Capriotti, ${ }^{56}$ A. Carbone, ${ }^{15, \mathrm{~h}}$ G. Carboni, ${ }^{25, \mathrm{i}}$ R. Cardinale, ${ }^{20, \mathrm{j}}$ A. Cardini, ${ }^{16}$ P. Carniti, ${ }^{21, \mathrm{~d}}$ L. Carson, ${ }^{52}$ K. Carvalho Akiba, ${ }^{2}$ G. Casse,${ }^{54}$ L. Cassina, ${ }^{21}$ M. Cattaneo, ${ }^{40}$ G. Cavallero, ${ }^{20,40, j}$ R. Cenci, ${ }^{24, k}$ D. Chamont, ${ }^{7}$ M. G. Chapman, ${ }^{48}$ M. Charles, ${ }^{8}$

Ph. Charpentier, ${ }^{40}$ G. Chatzikonstantinidis, ${ }^{47}$ M. Chefdeville, ${ }^{4}$ S. Chen, ${ }^{16}$ S. F. Cheung, ${ }^{57}$ S.-G. Chitic, ${ }^{40}$ V. Chobanova, ${ }^{39}$ M. Chrzaszcz, ${ }^{42}$ A. Chubykin, ${ }^{31}$ P. Ciambrone, ${ }^{19}$ X. Cid Vidal, ${ }^{39}$ G. Ciezarek,${ }^{40}$ P. E. L. Clarke, ${ }^{52}$ M. Clemencic, ${ }^{40}$ H. V. Cliff, ${ }^{49}$ J. Closier, ${ }^{40}$ V. Coco, ${ }^{40}$ J. Cogan, ${ }^{6}$ E. Cogneras, ${ }^{5}$ V. Cogoni, ${ }^{16, g}$ L. Cojocariu, ${ }^{30}$ P. Collins,${ }^{40}$ T. Colombo ${ }^{40}$ A. Comerma-Montells, ${ }^{12}$ A. Contu, ${ }^{16}$ G. Coombs, ${ }^{40}$ S. Coquereau, ${ }^{38}$ G. Corti,${ }^{40}$ M. Corvo, ${ }^{17, a}$ C. M. Costa Sobral, ${ }^{50}$ B. Couturier, ${ }^{40}$ G. A. Cowan, ${ }^{52}$ D. C. Craik, ${ }^{58}$ A. Crocombe, ${ }^{50}$ M. Cruz Torres, ${ }^{1}$ R. Currie,${ }^{52}$ C. D'Ambrosio, ${ }^{40}$ F. Da Cunha Marinho, ${ }^{2}$ C. L. Da Silva,${ }^{73}$ E. Dall'Occo, ${ }^{43}$ J. Dalseno, ${ }^{48}$ A. Davis, ${ }^{3}$ O. De Aguiar Francisco, ${ }^{40}$ K. De Bruyn,${ }^{40}$ S. De Capua ${ }^{56}$ M. De Cian, ${ }^{12}$ J. M. De Miranda, ${ }^{1}$ L. De Paula,${ }^{2}$ M. De Serio,${ }^{14,1}$ P. De Simone,${ }^{19}$ C. T. Dean, ${ }^{53}$ D. Decamp,${ }^{4}$ L. Del Buono, ${ }^{8}$ H.-P. Dembinski, ${ }^{11}$ M. Demmer, ${ }^{10}$ A. Dendek,${ }^{28}$ D. Derkach, ${ }^{35}$ O. Deschamps,${ }^{5}$ F. Dettori, ${ }^{54}$ B. Dey, ${ }^{65}$ A. Di Canto, ${ }^{40}$ P. Di Nezza, ${ }^{19}$ H. Dijkstra, ${ }^{40}$ F. Dordei, ${ }^{40}$ M. Dorigo, ${ }^{40}$ A. Dosil Suárez, ${ }^{39}$ L. Douglas, ${ }^{53}$ A. Dovbnya, ${ }^{45}$ 
K. Dreimanis, ${ }^{54}$ L. Dufour, ${ }^{43}$ G. Dujany, ${ }^{8}$ P. Durante, ${ }^{40}$ J. M. Durham, ${ }^{73}$ D. Dutta, ${ }^{56}$ R. Dzhelyadin, ${ }^{37}$ M. Dziewiecki, ${ }^{12}$ A. Dziurda, ${ }^{40}$ A. Dzyuba, ${ }^{31}$ S. Easo, ${ }^{51}$ U. Egede,${ }^{55}$ V. Egorychev, ${ }^{32}$ S. Eidelman, ${ }^{36, f}$ S. Eisenhardt, ${ }^{52}$ U. Eitschberger, ${ }^{10}$ R. Ekelhof, ${ }^{10}$ L. Eklund ${ }^{53}$ S. Ely, ${ }^{61}$ S. Esen, ${ }^{12}$ H. M. Evans, ${ }^{49}$ T. Evans, ${ }^{57}$ A. Falabella, ${ }^{15}$ N. Farley, ${ }^{47}$ S. Farry, ${ }^{54}$

D. Fazzini, ${ }^{21, d}$ L. Federici, ${ }^{25}$ D. Ferguson, ${ }^{52}$ G. Fernandez ${ }^{38}$ P. Fernandez Declara, ${ }^{40}$ A. Fernandez Prieto, ${ }^{39}$ F. Ferrari, ${ }^{15}$ L. Ferreira Lopes, ${ }^{41}$ F. Ferreira Rodrigues, ${ }^{2}$ M. Ferro-Luzzi, ${ }^{40}$ S. Filippov,${ }^{34}$ R. A. Fini,${ }^{14}$ M. Fiorini, ${ }^{17, a}$ M. Firlej, ${ }^{28}$ C. Fitzpatrick, ${ }^{41}$ T. Fiutowski, ${ }^{28}$ F. Fleuret, ${ }^{7, \mathrm{c}}$ M. Fontana,${ }^{16,40}$ F. Fontanelli, ${ }^{20, j}$ R. Forty, ${ }^{40}$ V. Franco Lima, ${ }^{54}$ M. Frank,${ }^{40}$ C. Frei, ${ }^{40}$ J. Fu, ${ }^{22, \mathrm{~m}}$ W. Funk ${ }^{40}$ E. Furfaro, ${ }^{25, \mathrm{i}}$ C. Färber, ${ }^{40}$ E. Gabriel,${ }^{52}$ A. Gallas Torreira, ${ }^{39}$ D. Galli, ${ }^{15, \mathrm{~h}}$ S. Gallorini,${ }^{23}$ S. Gambetta, ${ }^{52}$ M. Gandelman, ${ }^{2}$ P. Gandini, ${ }^{22}$ Y. Gao, ${ }^{3}$ L. M. Garcia Martin, ${ }^{71}$ J. García Pardiñas, ${ }^{39}$ J. Garra Tico, ${ }^{49}$ L. Garrido, ${ }^{38}$ D. Gascon, ${ }^{38}$ C. Gaspar,${ }^{40}$ L. Gavardi, ${ }^{10}$ G. Gazzoni, ${ }^{5}$ D. Gerick ${ }^{12}$ E. Gersabeck, ${ }^{56}$ M. Gersabeck, ${ }^{56}$ T. Gershon, ${ }^{50} \mathrm{Ph}$. Ghez,${ }^{4}$ S. Gianì ${ }^{41}$ V. Gibson, ${ }^{49}$ O. G. Girard ${ }^{41}$ L. Giubega, ${ }^{30}$ K. Gizdov, ${ }^{52}$ V. V. Gligorov, ${ }^{8}$ D. Golubkov, ${ }^{32}$ A. Golutvin,${ }^{55,69}$ A. Gomes, ${ }^{1, n}$ I. V. Gorelov, ${ }^{33}$ C. Gotti, ${ }^{21, d}$ E. Govorkova, ${ }^{43}$ J. P. Grabowski, ${ }^{12}$ R. Graciani Diaz, ${ }^{38}$ L. A. Granado Cardoso, ${ }^{40}$ E. Graugés,${ }^{38}$ E. Graverini, ${ }^{42}$ G. Graziani, ${ }^{18}$ A. Grecu, ${ }^{30}$ R. Greim, ${ }^{9}$ P. Griffith, ${ }^{16}$ L. Grillo, ${ }^{56}$ L. Gruber, ${ }^{40}$ B. R. Gruberg Cazon, ${ }^{57}$ O. Grünberg, ${ }^{67}$ E. Gushchin, ${ }^{34}$ Yu. Guz,${ }^{37}$ T. Gys, ${ }^{40}$ C. Göbel,${ }^{62}$ T. Hadavizadeh, ${ }^{57}$ C. Hadjivasiliou, ${ }^{5}$ G. Haefeli, ${ }^{41}$ C. Haen ${ }^{40}$ S. C. Haines,${ }^{49}$ B. Hamilton ${ }^{60}$ X. Han, ${ }^{12}$ T. H. Hancock, ${ }^{57}$

S. Hansmann-Menzemer, ${ }^{12}$ N. Harnew ${ }^{57}$ S. T. Harnew, ${ }^{48}$ C. Hasse, ${ }^{40}$ M. Hatch, ${ }^{40}$ J. He, ${ }^{63}$ M. Hecker, ${ }^{55}$ K. Heinicke, ${ }^{10}$ A. Heister, ${ }^{9}$ K. Hennessy, ${ }^{54}$ P. Henrard, ${ }^{5}$ L. Henry, ${ }^{71}$ E. van Herwijnen, ${ }^{40}$ M. Heß, ${ }^{67}$ A. Hicheur, ${ }^{2}$ D. Hill, ${ }^{57}$ P. H. Hopchev, ${ }^{41}$ W. Hu, ${ }^{65}$ W. Huang ${ }^{63}$ Z. C. Huard, ${ }^{59}$ W. Hulsbergen, ${ }^{43}$ T. Humair, ${ }^{55}$ M. Hushchyn, ${ }^{35}$ D. Hutcheroft, ${ }^{54}$ P. Ibis, ${ }^{10}$ M. Idzik ${ }^{28}$

P. Ilten, ${ }^{47}$ R. Jacobsson, ${ }^{40}$ J. Jalocha, ${ }^{57}$ E. Jans,${ }^{43}$ A. Jawahery, ${ }^{60}$ F. Jiang, ${ }^{3}$ M. John, ${ }^{57}$ D. Johnson, ${ }^{40}$ C. R. Jones, ${ }^{49}$ C. Joram, ${ }^{40}$ B. Jost ${ }^{40}$ N. Jurik, ${ }^{57}$ S. Kandybei, ${ }^{45}$ M. Karacson,${ }^{40}$ J. M. Kariuki ${ }^{48}$ S. Karodia, ${ }^{53}$ N. Kazeev,${ }^{35}$ M. Kecke, ${ }^{12}$ F. Keizer, ${ }^{49}$ M. Kelsey, ${ }^{61}$ M. Kenzie, ${ }^{49}$ T. Ketel,,${ }^{44}$ E. Khairullin, ${ }^{35}$ B. Khanji, ${ }^{12}$ C. Khurewathanakul, ${ }^{41}$ K. E. Kim, ${ }^{61}$ T. Kirn, ${ }^{9}$ S. Klaver, ${ }^{19}$ K. Klimaszewski, ${ }^{29}$ T. Klimkovich, ${ }^{11}$ S. Koliiev, ${ }^{46}$ M. Kolpin, ${ }^{12}$ R. Kopecna, ${ }^{12}$ P. Koppenburg, ${ }^{43}$

A. Kosmyntseva, ${ }^{32}$ S. Kotriakhova, ${ }^{31}$ M. Kozeiha, ${ }^{5}$ L. Kravchuk,${ }^{34}$ M. Kreps,${ }^{50}$ F. Kress, ${ }^{55}$ P. Krokovny, ${ }^{36, f}$ W. Krzemien, ${ }^{29}$

W. Kucewicz, ${ }^{27,0}$ M. Kucharczyk, ${ }^{27}$ V. Kudryavtsev, ${ }^{36, f}$ A. K. Kuonen ${ }^{41}$ T. Kvaratskheliya, ${ }^{32,40}$ D. Lacarrere, ${ }^{40}$

G. Lafferty, ${ }^{56}$ A. Lai, ${ }^{16}$ G. Lanfranchi,${ }^{19}$ C. Langenbruch, ${ }^{9}$ T. Latham,${ }^{50}$ C. Lazzeroni, ${ }^{47}$ R. Le Gac, ${ }^{6}$ A. Leflat,${ }^{33,40}$ J. Lefrançois, ${ }^{7}$ R. Lefèvre, ${ }^{5}$ F. Lemaitre, ${ }^{40}$ E. Lemos Cid, ${ }^{39}$ O. Leroy, ${ }^{6}$ T. Lesiak, ${ }^{27}$ B. Leverington, ${ }^{12}$ P.-R. Li, ${ }^{63}$ T. Li,${ }^{3}$ Y. Li ${ }^{7}$ Z. Li, ${ }^{61}$ X. Liang, ${ }^{61}$ T. Likhomanenko, ${ }^{68}$ R. Lindner, ${ }^{40}$ F. Lionetto, ${ }^{42}$ V. Lisovskyi, ${ }^{7}$ X. Liu, ${ }^{3}$ D. Loh, ${ }^{50}$ A. Loi, ${ }^{16}$

I. Longstaff, ${ }^{53}$ J. H. Lopes, ${ }^{2}$ D. Lucchesi, ${ }^{23, p}$ M. Lucio Martinez, ${ }^{39}$ H. Luo, ${ }^{52}$ A. Lupato, ${ }^{23}$ E. Luppi, ${ }^{17, a}$ O. Lupton, ${ }^{40}$ A. Lusiani, ${ }^{24}$ X. Lyu ${ }^{63}$ F. Machefert, ${ }^{7}$ F. Maciuc ${ }^{30}$ V. Macko, ${ }^{41}$ P. Mackowiak, ${ }^{10}$ S. Maddrell-Mander, ${ }^{48}$ O. Maev, ${ }^{31,40}$ K. Maguire,${ }^{56}$ D. Maisuzenko, ${ }^{31}$ M. W. Majewski,${ }^{28}$ S. Malde ${ }^{57}$ B. Malecki, ${ }^{27}$ A. Malinin, ${ }^{68}$ T. Maltsev,${ }^{36, f}$ G. Manca,,${ }^{16, g}$ G. Mancinelli, ${ }^{6}$ D. Marangotto, ${ }^{22, \mathrm{~m}}$ J. Maratas, ${ }^{5, \mathrm{q}}$ J. F. Marchand, ${ }^{4}$ U. Marconi,${ }^{15}$ C. Marin Benito, ${ }^{38}$ M. Marinangeli, ${ }^{41}$ P. Marino, ${ }^{41}$ J. Marks, ${ }^{12}$ G. Martellotti, ${ }^{26}$ M. Martin, ${ }^{6}$ M. Martinelli, ${ }^{41}$ D. Martinez Santos, ${ }^{39}$ F. Martinez Vidal, ${ }^{71}$ A. Massafferri, ${ }^{1}$ R. Matev ${ }^{40}$ A. Mathad,${ }^{50}$ Z. Mathe ${ }^{40}$ C. Matteuzzi, ${ }^{21}$ A. Mauri, ${ }^{42}$ E. Maurice,${ }^{7, c}$ B. Maurin, ${ }^{41}$

A. Mazurov, ${ }^{47}$ M. McCann,${ }^{55,40}$ A. McNab,${ }^{56}$ R. McNulty, ${ }^{13}$ J. V. Mead, ${ }^{54}$ B. Meadows, ${ }^{59}$ C. Meaux, ${ }^{6}$ F. Meier, ${ }^{10}$ N. Meinert, ${ }^{67}$ D. Melnychuk, ${ }^{29}$ M. Merk, ${ }^{43}$ A. Merli, ${ }^{22,40, m}$ E. Michielin, ${ }^{23}$ D. A. Milanes, ${ }^{66}$ E. Millard, ${ }^{50}$ M.-N. Minard, ${ }^{4}$ L. Minzoni, ${ }^{17}$ D. S. Mitzel, ${ }^{12}$ A. Mogini, ${ }^{8}$ J. Molina Rodriguez, ${ }^{1}$ T. Mombächer, ${ }^{10}$ I. A. Monroy, ${ }^{66}$ S. Monteil, ${ }^{5}$ M. Morandin, ${ }^{23}$ M. J. Morello, ${ }^{24, k}$ O. Morgunova, ${ }^{68}$ J. Moron, ${ }^{28}$ A. B. Morris,${ }^{52}$ R. Mountain, ${ }^{61}$ F. Muheim, ${ }^{52}$ M. Mulder ${ }^{43}$ D. Müller, ${ }^{40}$ J. Müller, ${ }^{10}$ K. Müller, ${ }^{42}$ V. Müller, ${ }^{10}$ P. Naik, ${ }^{48}$ T. Nakada, ${ }^{41}$ R. Nandakumar, ${ }^{51}$ A. Nandi,${ }^{57}$ I. Nasteva, ${ }^{2}$ M. Needham, ${ }^{52}$ N. Neri, ${ }^{22,40}$ S. Neubert, ${ }^{12}$ N. Neufeld ${ }^{40}$ M. Neuner, ${ }^{12}$ T. D. Nguyen, ${ }^{41}$ C. Nguyen-Mau, ${ }^{41, \mathrm{r}}$ S. Nieswand, ${ }^{9}$ R. Niet, ${ }^{10}$ N. Nikitin, ${ }^{33}$ T. Nikodem, ${ }^{12}$ A. Nogay, ${ }^{68}$ D. P. O'Hanlon, ${ }^{50}$ A. Oblakowska-Mucha, ${ }^{28}$ V. Obraztsov, ${ }^{37}$ S. Ogilvy, ${ }^{19}$ R. Oldeman, ${ }^{16, g}$ C. J. G. Onderwater, ${ }^{72}$ A. Ossowska, ${ }^{27}$ J. M. Otalora Goicochea, ${ }^{2}$ P. Owen, ${ }^{42}$ A. Oyanguren,${ }^{71}$ P. R. Pais, ${ }^{41}$ A. Palano, ${ }^{14}$ M. Palutan, ${ }^{19,40}$ G. Panshin,${ }^{70}$ A. Papanestis, ${ }^{51}$ M. Pappagallo, ${ }^{52}$ L. L. Pappalardo, ${ }^{17, a}$ W. Parker, ${ }^{60}$ C. Parkes,${ }^{56}$ G. Passaleva, ${ }^{18,40}$ A. Pastore, ${ }^{14,1}$ M. Patel,${ }^{55}$ C. Patrignani, ${ }^{15, \mathrm{~h}}$ A. Pearce,${ }^{40}$ A. Pellegrino, ${ }^{43}$ G. Penso, ${ }^{26}$ M. Pepe Altarelli, ${ }^{40}$ S. Perazzini, ${ }^{40}$ D. Pereima, ${ }^{32}$ P. Perret, ${ }^{5}$ L. Pescatore ${ }^{41}$ K. Petridis, ${ }^{48}$ A. Petrolini, ${ }^{20, j}$ A. Petrov,${ }^{68}$ M. Petruzzo, ${ }^{22, m}$

E. Picatoste Olloqui, ${ }^{38}$ B. Pietrzyk, ${ }^{4}$ G. Pietrzyk,${ }^{41}$ M. Pikies, ${ }^{27}$ D. Pinci, ${ }^{26}$ F. Pisani, ${ }^{40}$ A. Pistone, ${ }^{20, j}$ A. Piucci, ${ }^{12}$ V. Placinta, ${ }^{30}$ S. Playfer, ${ }^{52}$ M. Plo Casasus, ${ }^{39}$ F. Polci, ${ }^{8}$ M. Poli Lener, ${ }^{19}$ A. Poluektov,${ }^{50}$ I. Polyakov, ${ }^{61}$ E. Polycarpo, ${ }^{2}$ G. J. Pomery, ${ }^{48}$ S. Ponce,${ }^{40}$ A. Popov,${ }^{37}$ D. Popov,,${ }^{11,40}$ S. Poslavskii, ${ }^{37}$ C. Potterat, ${ }^{2}$ E. Price, ${ }^{48}$ J. Prisciandaro,${ }^{39}$ C. Prouve, ${ }^{48}$ V. Pugatch, ${ }^{46}$ A. Puig Navarro, ${ }^{42}$ H. Pullen, ${ }^{57}$ G. Punzi, ${ }^{24, s}$ W. Qian, ${ }^{50}$ J. Qin, ${ }^{63}$ R. Quagliani, ${ }^{8}$ B. Quintana, ${ }^{5}$ B. Rachwal, ${ }^{28}$ J. H. Rademacker, ${ }^{48}$ M. Rama, ${ }^{24}$ M. Ramos Pernas, ${ }^{39}$ M. S. Rangel, ${ }^{2}$ I. Raniuk, ${ }^{45}$ F. Ratnikov, ${ }^{35, t}$ G. Raven, ${ }^{44}$ M. Ravonel Salzgeber, ${ }^{40}$ M. Reboud, ${ }^{4}$ F. Redi, ${ }^{41}$ S. Reichert, ${ }^{10}$ A. C. dos Reis, ${ }^{1}$ C. Remon Alepuz, ${ }^{71}$ V. Renaudin, ${ }^{7}$ 
S. Ricciardi, ${ }^{51}$ S. Richards ${ }^{48}$ M. Rihl,${ }^{40}$ K. Rinnert, ${ }^{54}$ P. Robbe, ${ }^{7}$ A. Robert, ${ }^{8}$ A. B. Rodrigues,${ }^{41}$ E. Rodrigues,${ }^{59}$ J. A. Rodriguez Lopez, ${ }^{66}$ A. Rogozhnikov, ${ }^{35}$ S. Roiser, ${ }^{40}$ A. Rollings, ${ }^{57}$ V. Romanovskiy, ${ }^{37}$ A. Romero Vidal, ${ }^{39,40}$ M. Rotondo, ${ }^{19}$ M. S. Rudolph, ${ }^{61}$ T. Ruf, ${ }^{40}$ P. Ruiz Valls, ${ }^{71}$ J. Ruiz Vidal, ${ }^{71}$ J. J. Saborido Silva, ${ }^{39}$ E. Sadykhov, ${ }^{32}$ N. Sagidova, ${ }^{31}$ B. Saitta, ${ }^{16, \mathrm{~g}}$ V. Salustino Guimaraes ${ }^{62}$ C. Sanchez Mayordomo, ${ }^{71}$ B. Sanmartin Sedes, ${ }^{39}$ R. Santacesaria, ${ }^{26}$ C. Santamarina Rios, ${ }^{39}$ M. Santimaria, ${ }^{19}$ E. Santovetti, ${ }^{25, i}$ G. Sarpis, ${ }^{56}$ A. Sarti, ${ }^{19, u}$ C. Satriano, ${ }^{26, v}$ A. Satta, ${ }^{25}$ D. M. Saunders ${ }^{48}$ D. Savrina,${ }^{32,33}$ S. Schael, ${ }^{9}$ M. Schellenberg, ${ }^{10}$ M. Schiller, ${ }^{53}$ H. Schindler,${ }^{40}$ M. Schmelling, ${ }^{11}$ T. Schmelzer, ${ }^{10}$ B. Schmidt ${ }^{40}$ O. Schneider, ${ }^{41}$ A. Schopper, ${ }^{40}$ H. F. Schreiner, ${ }^{59}$ M. Schubiger, ${ }^{41}$ M. H. Schune, ${ }^{7}$ R. Schwemmer, ${ }^{40}$ B. Sciascia, ${ }^{19}$ A. Sciubba, ${ }^{26, u}$ A. Semennikov, ${ }^{32}$ E. S. Sepulveda, ${ }^{8}$ A. Sergi, ${ }^{47}$ N. Serra, ${ }^{42}$ J. Serrano, ${ }^{6}$ L. Sestini, ${ }^{23}$ P. Seyfert, ${ }^{40}$ M. Shapkin, ${ }^{37}$ Y. Shcheglov, ${ }^{31}$ T. Shears, ${ }^{54}$ L. Shekhtman, ${ }^{36, f}$ V. Shevchenko, ${ }^{68}$ B. G. Siddi,${ }^{17}$ R. Silva Coutinho, ${ }^{42}$ L. Silva de Oliveira, ${ }^{2}$ G. Simi ${ }^{23, p}$ S. Simone, ${ }^{14,1}$ M. Sirendi, ${ }^{49}$ N. Skidmore, ${ }^{48}$ T. Skwarnicki, ${ }^{61}$ I. T. Smith, ${ }^{52}$ J. Smith, ${ }^{49}$ M. Smith, ${ }^{55}$ 1. Soares Lavra, ${ }^{1}$ M. D. Sokoloff ${ }^{59}$ F. J. P. Soler, ${ }^{53}$ B. Souza De Paula, ${ }^{2}$ B. Spaan, ${ }^{10}$ P. Spradlin,${ }^{53}$ F. Stagni, ${ }^{40}$ M. Stahl, ${ }^{12}$ S. Stahl,${ }^{40}$ P. Stefko, ${ }^{41}$ S. Stefkova, ${ }^{55}$ O. Steinkamp, ${ }^{42}$ S. Stemmle, ${ }^{12}$ O. Stenyakin,${ }^{37}$ M. Stepanova, ${ }^{31}$ H. Stevens, ${ }^{10}$ S. Stone, ${ }^{61}$ B. Storaci, ${ }^{42}$ S. Stracka, ${ }^{24, s}$ M. E. Stramaglia, ${ }^{41}$ M. Straticiuc, ${ }^{30}$ U. Straumann, ${ }^{42}$ S. Strokov, ${ }^{70}$ J. Sun, ${ }^{3}$ L. Sun,${ }^{64}$ K. Swientek, ${ }^{28}$ V. Syropoulos, ${ }^{44}$ T. Szumlak, ${ }^{28}$ M. Szymanski, ${ }^{63}$ S. T'Jampens, ${ }^{4}$ A. Tayduganov, ${ }^{6}$ T. Tekampe, ${ }^{10}$ G. Tellarini, ${ }^{17, a}$ F. Teubert, ${ }^{40}$ E. Thomas, ${ }^{40}$ J. van Tilburg, ${ }^{43}$ M. J. Tilley, ${ }^{55}$ V. Tisserand, ${ }^{5}$ M. Tobin, ${ }^{41}$ S. Tolk, ${ }^{49}$ L. Tomassetti, ${ }^{17, a}$ D. Tonelli, ${ }^{24}$ R. Tourinho Jadallah Aoude,,${ }^{1}$ E. Tournefier, ${ }^{4}$ M. Traill, ${ }^{53}$ M. T. Tran, ${ }^{41}$ M. Tresch, ${ }^{42}$ A. Trisovic, ${ }^{49}$ A. Tsaregorodtsev, ${ }^{6}$ P. Tsopelas, ${ }^{43}$ A. Tully, ${ }^{49}$ N. Tuning, ${ }^{43,40}$ A. Ukleja, ${ }^{29}$ A. Usachov, ${ }^{7}$ A. Ustyuzhanin,${ }^{35}$ U. Uwer, ${ }^{12}$ C. Vacca, ${ }^{16,9}$ A. Vagner ${ }^{70}$ V. Vagnoni,${ }^{15,40}$ A. Valassi ${ }^{40}$ S. Valat, ${ }^{40}$ G. Valenti, ${ }^{15}$ R. Vazquez Gomez ${ }^{40}$ P. Vazquez Regueiro, ${ }^{39}$ S. Vecchi, ${ }^{17}$ M. van Veghel, ${ }^{43}$ J. J. Velthuis,${ }^{48}$ M. Veltri, ${ }^{18, w}$ G. Veneziano, ${ }^{57}$ A. Venkateswaran, ${ }^{61}$ T. A. Verlage, ${ }^{9}$ M. Vernet, ${ }^{5}$ M. Vesterinen, ${ }^{57}$ J. V. Viana Barbosa ${ }^{40}$ D. Vieira, ${ }^{63}$ M. Vieites Diaz, ${ }^{39}$ H. Viemann, ${ }^{67}$ X. Vilasis-Cardona, ${ }^{38, b}$ M. Vitti, ${ }^{49}$ V. Volkov, ${ }^{33}$ A. Vollhardt, ${ }^{42}$ B. Voneki, ${ }^{40}$ A. Vorobyev, ${ }^{31}$ V. Vorobyev,,${ }^{36, f}$ C. Voß, ${ }^{9}$ J. A. de Vries, ${ }^{43}$ C. Vázquez Sierra, ${ }^{43}$ R. Waldi, ${ }^{67}$ J. Walsh, ${ }^{24}$ J. Wang, ${ }^{61}$ Y. Wang, ${ }^{65}$ D. R. Ward, ${ }^{49}$ H. M. Wark, ${ }^{54}$ N. K. Watson, ${ }^{47}$ D. Websdale, ${ }^{55}$ A. Weiden, ${ }^{42}$ C. Weisser, ${ }^{58}$ M. Whitehead,${ }^{40}$ J. Wicht, ${ }^{50}$ G. Wilkinson, ${ }^{57}$ M. Wilkinson, ${ }^{61}$ M. Williams, ${ }^{56}$ M. Williams, ${ }^{58}$ T. Williams, ${ }^{47}$ F. F. Wilson, ${ }^{51,40}$ J. Wimberley, ${ }^{60}$ M. Winn, ${ }^{7}$ J. Wishahi, ${ }^{10}$ W. Wislicki, ${ }^{29}$ M. Witek, ${ }^{27}$ G. Wormser, ${ }^{7}$ S. A. Wotton, ${ }^{49}$ K. Wyllie, ${ }^{40}$ Y. Xie, ${ }^{65}$ M. Xu, ${ }^{65}$ Q. Xu, ${ }^{63}$ Z. Xu, ${ }^{3}$ Z. Xu, ${ }^{4}$ Z. Yang, ${ }^{3}$ Z. Yang, ${ }^{60}$ Y. Yao, ${ }^{61}$ H. Yin ${ }^{65}$ J. Yu, ${ }^{65}$ X. Yuan, ${ }^{61}$ O. Yushchenko, ${ }^{37}$ K. A. Zarebski, ${ }^{47}$ M. Zavertyaev, ${ }^{11, x}$ L. Zhang, ${ }^{3}$ Y. Zhang, ${ }^{7}$ A. Zhelezov, ${ }^{12}$ Y. Zheng, ${ }^{63}$ X. Zhu, ${ }^{3}$ V. Zhukov, ${ }^{9,33}$ J. B. Zonneveld, ${ }^{52}$ and S. Zucchelli ${ }^{15}$

\title{
(LHCb Collaboration)
}

\author{
${ }^{1}$ Centro Brasileiro de Pesquisas Físicas $(C B P F)$, Rio de Janeiro, Brazil \\ ${ }^{2}$ Universidade Federal do Rio de Janeiro (UFRJ), Rio de Janeiro, Brazil \\ ${ }^{3}$ Center for High Energy Physics, Tsinghua University, Beijing, China \\ ${ }^{4}$ Univ. Grenoble Alpes, Univ. Savoie Mont Blanc, CNRS, IN2P3-LAPP, Annecy, France \\ ${ }^{5}$ Clermont Université, Université Blaise Pascal, CNRS/IN2P3, LPC, Clermont-Ferrand, France \\ ${ }^{6}$ Aix Marseille Univ, CNRS/IN2P3, CPPM, Marseille, France \\ ${ }^{7}$ LAL, Univ. Paris-Sud, CNRS/IN2P3, Université Paris-Saclay, Orsay, France \\ ${ }^{8}$ LPNHE, Université Pierre et Marie Curie, Université Paris Diderot, CNRS/IN2P3, Paris, France \\ ${ }^{9}$ I. Physikalisches Institut, RWTH Aachen University, Aachen, Germany \\ ${ }^{10}$ Fakultät Physik, Technische Universität Dortmund, Dortmund, Germany \\ ${ }^{11}$ Max-Planck-Institut für Kernphysik (MPIK), Heidelberg, Germany \\ ${ }^{12}$ Physikalisches Institut, Ruprecht-Karls-Universität Heidelberg, Heidelberg, Germany \\ ${ }^{13}$ School of Physics, University College Dublin, Dublin, Ireland \\ ${ }^{14}$ Sezione INFN di Bari, Bari, Italy \\ ${ }^{15}$ Sezione INFN di Bologna, Bologna, Italy \\ ${ }^{16}$ Sezione INFN di Cagliari, Cagliari, Italy \\ ${ }^{17}$ Universita e INFN, Ferrara, Ferrara, Italy \\ ${ }^{18}$ Sezione INFN di Firenze, Firenze, Italy \\ ${ }^{19}$ Laboratori Nazionali dell'INFN di Frascati, Frascati, Italy \\ ${ }^{20}$ Sezione INFN di Genova, Genova, Italy \\ ${ }^{21}$ Sezione INFN di Milano Bicocca, Milano, Italy \\ ${ }^{22}$ Sezione di Milano, Milano, Italy
}




\footnotetext{
${ }^{23}$ Sezione INFN di Padova, Padova, Italy

${ }^{24}$ Sezione INFN di Pisa, Pisa, Italy

${ }^{25}$ Sezione INFN di Roma Tor Vergata, Roma, Italy

${ }^{26}$ Sezione INFN di Roma La Sapienza, Roma, Italy

${ }^{27}$ Henryk Niewodniczanski Institute of Nuclear Physics Polish Academy of Sciences, Kraków, Poland

${ }^{28}$ AGH-University of Science and Technology, Faculty of Physics and Applied Computer Science, Kraków, Poland

${ }^{29}$ National Center for Nuclear Research (NCBJ), Warsaw, Poland

${ }^{30}$ Horia Hulubei National Institute of Physics and Nuclear Engineering, Bucharest-Magurele, Romania

${ }^{31}$ Petersburg Nuclear Physics Institute (PNPI), Gatchina, Russia

${ }^{32}$ Institute of Theoretical and Experimental Physics (ITEP), Moscow, Russia

${ }^{33}$ Institute of Nuclear Physics, Moscow State University (SINP MSU), Moscow, Russia

${ }^{34}$ Institute for Nuclear Research of the Russian Academy of Sciences (INR RAS), Moscow, Russia

${ }^{35}$ Yandex School of Data Analysis, Moscow, Russia

${ }^{36}$ Budker Institute of Nuclear Physics (SB RAS), Novosibirsk, Russia

${ }^{37}$ Institute for High Energy Physics (IHEP), Protvino, Russia

${ }^{38}$ ICCUB, Universitat de Barcelona, Barcelona, Spain

${ }^{39}$ Instituto Galego de Física de Altas Enerxías (IGFAE), Universidade de Santiago de Compostela, Santiago de Compostela, Spain

${ }^{40}$ European Organization for Nuclear Research (CERN), Geneva, Switzerland

${ }^{41}$ Institute of Physics, Ecole Polytechnique Fédérale de Lausanne (EPFL), Lausanne, Switzerland

${ }^{42}$ Physik-Institut, Universität Zürich, Zürich, Switzerland

${ }^{43}$ Nikhef National Institute for Subatomic Physics, Amsterdam, Netherlands

${ }^{44}$ Nikhef National Institute for Subatomic Physics and VU University Amsterdam, Amsterdam, Netherlands

${ }^{45}$ NSC Kharkiv Institute of Physics and Technology (NSC KIPT), Kharkiv, Ukraine

${ }^{46}$ Institute for Nuclear Research of the National Academy of Sciences (KINR), Kyiv, Ukraine

${ }^{47}$ University of Birmingham, Birmingham, United Kingdom

${ }^{48}$ H.H. Wills Physics Laboratory, University of Bristol, Bristol, United Kingdom

${ }^{49}$ Cavendish Laboratory, University of Cambridge, Cambridge, United Kingdom

${ }^{50}$ Department of Physics, University of Warwick, Coventry, United Kingdom

${ }^{51}$ STFC Rutherford Appleton Laboratory, Didcot, United Kingdom

${ }^{52}$ School of Physics and Astronomy, University of Edinburgh, Edinburgh, United Kingdom

${ }^{53}$ School of Physics and Astronomy, University of Glasgow, Glasgow, United Kingdom

${ }^{54}$ Oliver Lodge Laboratory, University of Liverpool, Liverpool, United Kingdom

${ }^{55}$ Imperial College London, London, United Kingdom

${ }^{56}$ School of Physics and Astronomy, University of Manchester, Manchester, United Kingdom

${ }^{57}$ Department of Physics, University of Oxford, Oxford, United Kingdom

${ }^{58}$ Massachusetts Institute of Technology, Cambridge, Massachusetts, USA

${ }^{59}$ University of Cincinnati, Cincinnati, Ohio, USA

${ }^{60}$ University of Maryland, College Park, Maryland, USA

${ }^{61}$ Syracuse University, Syracuse, New York, USA

${ }^{62}$ Pontifícia Universidade Católica do Rio de Janeiro (PUC-Rio), Rio de Janeiro, Brazil

(associated with Institution Universidade Federal do Rio de Janeiro (UFRJ), Rio de Janeiro, Brazil)

${ }^{63}$ University of Chinese Academy of Sciences, Beijing, China

(associated with Institution Center for High Energy Physics, Tsinghua University, Beijing, China)

${ }^{64}$ School of Physics and Technology, Wuhan University, Wuhan, China

(associated with Institution Center for High Energy Physics, Tsinghua University, Beijing, China)

${ }^{65}$ Institute of Particle Physics, Central China Normal University, Wuhan, Hubei, China

(associated with Institution Center for High Energy Physics, Tsinghua University, Beijing, China)

${ }^{66}$ Departamento de Fisica, Universidad Nacional de Colombia, Bogota, Colombia

(associated with Institution LPNHE, Université Pierre et Marie Curie, Université Paris Diderot, CNRS/IN2P3, Paris, France)

${ }^{67}$ Institut für Physik, Universität Rostock, Rostock, Germany

(associated with Institution Physikalisches Institut, Ruprecht-Karls-Universität Heidelberg, Heidelberg, Germany)

${ }^{68}$ National Research Centre Kurchatov Institute, Moscow, Russia

(associated with Institution Institute of Theoretical and Experimental Physics (ITEP), Moscow, Russia)

${ }^{69}$ National University of Science and Technology MISIS, Moscow, Russia

(associated with Institution Institute of Theoretical and Experimental Physics (ITEP), Moscow, Russia)

${ }^{70}$ National Research Tomsk Polytechnic University, Tomsk, Russia

(associated with Institution Institute of Theoretical and Experimental Physics (ITEP), Moscow, Russia)

${ }^{71}$ Instituto de Fisica Corpuscular, Centro Mixto Universidad de Valencia-CSIC, Valencia, Spain

(associated with Institution ICCUB, Universitat de Barcelona, Barcelona, Spain)
} 


\section{${ }^{72}$ Van Swinderen Institute, University of Groningen, Groningen, Netherlands \\ (associated with Institution Nikhef National Institute for Subatomic Physics, Amsterdam, Netherlands) \\ ${ }^{73}$ Los Alamos National Laboratory (LANL), Los Alamos, United States \\ (associated with Institution Syracuse University, Syracuse, New York, USA)}

${ }^{a}$ Also at Università di Ferrara, Ferrara, Italy.

${ }^{\mathrm{b}}$ Also at LIFAELS, La Salle, Universitat Ramon Llull, Barcelona, Spain.

${ }^{c}$ Also at Laboratoire Leprince-Ringuet, Palaiseau, France.

${ }^{\mathrm{d}}$ Also at Università di Milano Bicocca, Milano, Italy.

${ }^{\mathrm{e}}$ Also at Università di Modena e Reggio Emilia, Modena, Italy.

${ }^{\mathrm{f}}$ Also at Novosibirsk State University, Novosibirsk, Russia.

${ }^{\mathrm{g}}$ Also at Università di Cagliari, Cagliari, Italy.

${ }^{\mathrm{h}}$ Also at Università di Bologna, Bologna, Italy.

${ }^{\mathrm{i}}$ Also at Università di Roma Tor Vergata, Roma, Italy.

${ }^{\mathrm{j}}$ Also at Università di Genova, Genova, Italy.

${ }^{\mathrm{k}}$ Also at Scuola Normale Superiore, Pisa, Italy.

${ }^{1}$ Also at Università di Bari, Bari, Italy.

${ }^{\mathrm{m}}$ Also at Università degli Studi di Milano, Milano, Italy.

${ }^{\mathrm{n}}$ Also at Universidade Federal do Triângulo Mineiro (UFTM), Uberaba-MG, Brazil.

${ }^{\circ}$ Also at AGH-University of Science and Technology, Faculty of Computer Science, Electronics and Telecommunications, Kraków, Poland.

${ }^{\mathrm{p}}$ Also at Università di Padova, Padova, Italy.

${ }^{\mathrm{q}}$ Also at Iligan Institute of Technology (IIT), Iligan, Philippines.

${ }^{\mathrm{r}}$ Also at Hanoi University of Science, Hanoi, Vietnam.

${ }^{\mathrm{s}}$ Also at Università di Pisa, Pisa, Italy.

${ }^{t}$ Also at National Research University Higher School of Economics, Moscow, Russia.

"Also at Università di Roma La Sapienza, Roma, Italy.

${ }^{v}$ Also at Università della Basilicata, Potenza, Italy.

${ }^{\mathrm{w}}$ Also at Università di Urbino, Urbino, Italy.

${ }^{x}$ Also at P.N. Lebedev Physical Institute, Russian Academy of Science (LPI RAS), Moscow, Russia. 